\title{
Cov-19 Incidence Detection Using Efficient Intelligent Multi Agent System Based on Dynamic Unsupervised Feed-Forward Neural Network
}

\author{
S. Raviraja ${ }^{1 *}$, Roya Asadi ${ }^{2}$, Negin Kourosdari ${ }^{3}$, Kanthesh M Basalingappa ${ }^{4}$ and Hemashree $\mathrm{L}^{5}$ \\ ${ }^{1 *}$ University of Garden City, Khartoum, Sudan and PI, Royal Research Foundation, Karnataka, India \\ ${ }^{2}$ Department of Computer Engineering, Fouman Faculty of Engineering - The Campus \\ of the Faculty of Technology, University of Tehran, Iran. \\ ${ }^{3}$ Department of Electronic and Electrical Engineering, Shariaty Technical \\ and Vocational University, Tehran, Iran \\ ${ }^{4}$ Department of Molecular Science, Scholar JSS Academy of Higher Education and Research, India \\ ${ }^{5}$ Department of Biomedical Science, Scholar JSS Academy of Higher Education and Research, India
}

\section{ABSTRACT}

Nowadays Corona Virus Disease, that it has been breaking out since 2019 (COV-19) which is outset from Wuhan of China, is a critical problem to research in the world. COV-19 is consistently infecting wave after waves in a variety of symptomatic way. We propose an Intelligent Multi Agent Systems (IMAS) based on the dynamic unsupervised feed forward neural network (DUFFNN) model, which authors believe that even after vaccine inventions repeatedly the infections are spreading in terms of second wave and so on, it has great potentials to apply in detecting the current status of the COV-19 in the world and predict its future and prevent. The IMAS framework has two flows. First flow includes earning knowledge of the existing symptoms, diagnostic techniques and treatment of the deadly pandemic of the COV-19. Second flow includes detection of the number of patient cases, risk of the disease and the polluted spots, that they can help infected to monitor and control the COV-19 risk and parameters. The proposed experimental results show that the DUFFNN model and consequently its semi-clustering have the best efficient results 98.99\% and 99.97\% accuracy respectively, which are the best results in this area by comparison with different related methods.

KEY WORDS: ARTIFICIAL NEURAL NETWORK (ANN) MODEL, DYNAMIC UNSUPERVISED FEED FORWARD NEURAL NETWORK (DUFFNN) MODEL, INTELLIGENT MULTI AGENT SYSTEM (IMAS), CORONA VIRUS DISEASE 2019.

\section{INTRODUCTION}

In several applications of real time research domains, like banking mastercard transactions, multi agent intelligent systems, and medical informatics, use online nonstop data that are updated frequently. The casual occurrence of ordinary problems of such domains are assortment, memory storage, searching data transactions, data transfer, visualization and analysis of huge capacity of such data including data noise and therefore the high magnitude of the info. Data processing may be a

Biosc Biotech Res Comm P-ISSN: 0974-6455 E-ISSN: 2321-4007

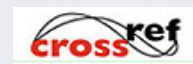

Identifiers and Pagination

Year: 2021 Vol: 14 No (5) Special Issue

Pages: $283-293$

This is an open access article under Creative

Commons License Attribn 4.0 Intl (CC-BY).

DOI: $h t t p: / / d x$.doi.org/10.21786/bbrc/14.5/51 technique of the knowledge detection process in databases (KDD), that's the techniques of analyzing the info, exploit the knowledge, potentially useful information supported the relationships between data values during a huge rational databases, recognizing meaningful patterns and rules out of very great deal of knowledge. Accordingly, clustering methods in data processing are not to identify the distinguish structure and determine the knowledge of the info, so as to group the specified data implicitly.

Considering the info area is a web non-stationary, the web Online Dynamic Unsupervised Feed-Forward Neural Network (ODUFFNN) clustering methods must support online incremental learning. In these environment, the info are often seems to be highly huge, continuous and uninterrupted input file, the distributions of knowledge aren't known and therefore the data distribution might modify over the period of time. Incremental learning signifies to the power of frequently training a network using new data or deleting unnecessary data, without

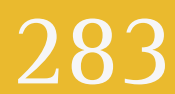


terminating outdated prototype patterns. The ODUFFNN clustering technique has capability to regulate the randomly fluctuating data, correct its algorithm and modify automatically during a feasible approach to new settings of the world over time dynamically for processing of both data and therefore the knowledge. The ODUFFNN capable to supply accommodations and crop the info and rules without terminating old knowledge, algorithm to coach variety of clusters and density of each cluster without predetermining the principles . Further, the ODUFFNN method must control time, memory space and accuracy efficiently.

The ODUFFNN clustering are founded on the Unsupervised Feed Forward Neural Network (UFFNN) clustering methods. The UFFNN clustering methods are usually apply Hebbian learning, or competitive learning, or the combined the both techniques. The similarities between Hebbian and competitive learning are unsupervised learning without error signal, and strongly related to biological systems. Though, competitive learning just one output must be active; such, only the winner weights are updated in each period of your time. With contrast, no constraint is imposed by neighboring nodes in Hebbian learning, and every one weight is updated at whenever period. Competitive learning uses vector quantization (VQ) through clustering. The static UFFNN clustering methods are supported the VQ and K-means. K-means clustering model partitions nodes supported the CentroidBased technique. Linde-Buzo-Gray algorithm (LBG) may be a VQ algorithm to realize an honest code-book and is probabilistic model on long training.

The VQ is applied for probability density functions modeling by the distribution of prototype vectors. The Growing Neural Gas (GNG) model and Self-Organization Map (SOM) are samples of the UFFNN clustering. The GNG method can follow dynamic distributions by adding nodes and deleting them within the network through clustering by using the function parameters. SOM maps multi-dimensional data onto lower dimensional subspaces, with the geometric affiliations between points indicates their similarity, on the idea of a competitive learning algorithm. The weights are adjusted and supported their proximity of the "winning" nodes, that's the foremost closely appear as if a sample input.

Table 1. Comparison of the DUFFNN clustering method with current ODUFFNN clustering methods

\begin{tabular}{|c|c|c|c|}
\hline $\begin{array}{l}\text { The ODUFFNN } \\
\text { method }\end{array}$ & The ESOM & The DSOM & The DUFFNN \\
\hline Essential forms & SOM and GNG, Hebbian & SOM & Hebbian and ESOM \\
\hline \multirow{11}{*}{$\begin{array}{c}\text { Several dauntless } \\
\text { features } \\
\text { (Advantages) }\end{array}$} & \multirow[b]{2}{*}{ Begin without any node } & \multirow[b]{2}{*}{$\begin{array}{c}\text { Improve the formula of updating } \\
\text { weights }\end{array}$} & Starts without any node \\
\hline & & & $\begin{array}{c}\text { Input vectors are not stored during } \\
\text { learning }\end{array}$ \\
\hline & $\begin{array}{l}\text { Automatic Update with } \\
\text { online input data }\end{array}$ & \multirow{7}{*}{ Elasticity or Flexibility property } & $\begin{array}{l}\text { Automatic Update with online input } \\
\text { data }\end{array}$ \\
\hline & $\begin{array}{l}\text { Operates the nodes with } \\
\text { weak }\end{array}$ & & Operates the nodes with weak \\
\hline & \multirow{5}{*}{$\begin{array}{l}\text { Possible to crop the } \\
\text { thresholds }\end{array}$} & & Possible to crop the thresholds \\
\hline & & & Initialize non-random weights \\
\hline & & & $\begin{array}{l}\text { Non-sensitive to the order of the data } \\
\text { entry }\end{array}$ \\
\hline & & & Mining $B M W$ \\
\hline & & & $\begin{array}{l}\text { Clustering during one epoch without } \\
\text { updating weights }\end{array}$ \\
\hline & \multirow{2}{*}{$\begin{array}{l}\text { Clustering during one } \\
\text { epoch }\end{array}$} & \multirow{2}{*}{ Earning Best Match Unit (BMU) } & Able to retrieve old data \\
\hline & & & Able to learn the number of clusters \\
\hline Time Complexity & $O\left(n^{2} \cdot m\right)$ & $O\left(\right.$ c.n. $\left.m^{2}\right)$ & $O\left(n^{2}\right)$ \\
\hline $\begin{array}{c}\text { Memory } \\
\text { Complexity }\end{array}$ & $O\left(n^{2} \cdot m \cdot s_{m}\right)$ & $O\left(c . n \cdot m^{2} \cdot s_{m}\right)$ & $O\left(n^{2} \cdot s_{m}\right)$ \\
\hline
\end{tabular}

Effective ODUFFNN clustering method frequently employ the competitive learning as applied within the dynamic self-organizing map (DSOM), or competitive Hebbian learning as utilized in the Evolving Self-Organizing Map (ESOM) [10]. ESOM is made on using SOM and GNG methods. ESOM starts without nodes. The network updates itself with online admission, and if required, it creates new nodes through one training epoch. Almost like SOM, each node features a special weight vector. The strong neighborhood relative is driven by the space between connected nodes, hence, it's sensitive to noise nodes, weak connections and isolated nodes built on
Hebbian learning. Therefore, if the space is just too big, it creates a weak threshold and therefore the connection are often pruned. DSOM is far almost like SOM supported competitive learning. So as to update the weights of the neighboring nodes, time dependency is detached, and therefore the parameter of the elasticity or flexibility is taken into account, which is learned by using trial and error. If the parameter of the resistance is just too high, DSOM doesn't assemble; and if it's too low, it's going to avoid DSOM to occur and isn't sensitive to the relation between neighbor nodes. 
If no node is close enough to the input values, other nodes must learn consistent with their distance to the input value. Also, the DUFFNN is an efficient single-layer method with one epoch training, data dimensionality reduction and therefore the capabilities to overwhelm the foremost declared problems, suitable to use within the communication network area. Within the DUFFNN, dynamically after admission of every new online input file, the code book of non-random and real weights and other important information about online data as essentially important information (EII) are updated and stored within the memory. Accordingly, the exclusion threshold of the info is calculated on the idea of the EII and therefore the data is trained. Then, the network of trained nodes is updated. The training process is predicated on considering a linear activation function and therefore the exclusive threshold.

Figure 1: Epidemic curve of COV-19 deaths, by date of report and WHO region until 28th March, 2021

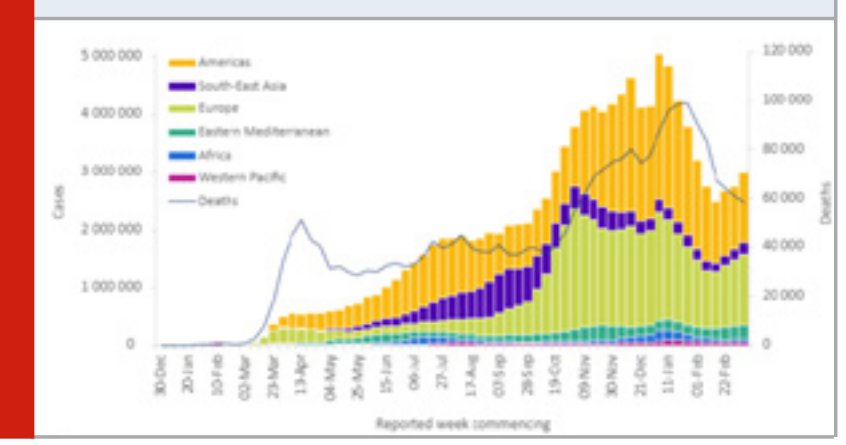

Figure 2: Outline of IMA based NN clustering system

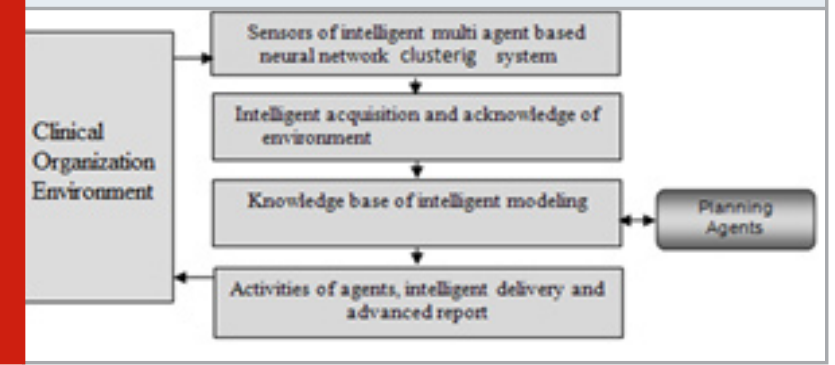

In Table 1, authors have planned to compare the DUFFNN clustering method with the ESOM and DSOM as affective current ODUFFNN clustering methods. With the respective algorithms, the time and memory complexities of the ESOM are O(n2.m) and O(n2.m.sm). The time and memory complexities of the DSOM are O(c.n.m2) and O(c.n.m2.sm). However, the DUFFNN method has better leads to the time and memory complexities which are $\mathrm{O}(\mathrm{n} 2)$ and $\mathrm{O}(\mathrm{n} 2 . \mathrm{sm})$. Where the parameters $\mathrm{c}, \mathrm{k}, \mathrm{n}, \mathrm{m}$, sm are the amount of epochs, clusters, nodes, attributes and size of every attribute, respectively. The observation above reveals that the DUFFNN method more efficient than compared to other related methods.

Several litterateurs dedicated to enhance the UFFNN and ODUFFNN methods by the technique of using constraints like class labels. The constraints of sophistication labels are supported the knowledge of experts and therefore the user guide as partial supervision for improved controlling the tasks of clustering and desired results. The Semi-ESOM and Dynamic Semi-supervised Feed Forward Neural Network (DSFFNN) clustering techniques are examples during this domain. so as to enhance the methods, many researchers manage and proper the amount of clusters and density of every cluster by adding and deleting the info nodes and clusters, thereafter clustering, the models assigned class label to the winning node and accordingly assigned an equivalent class labels to its neighbor nodes in its cluster. Each cluster must have a singular class label, if the info nodes of a cluster have different class labels, the cluster is in a position to be divided into different sub-clusters.

Though, assigning the category labels to the info nodes between the clusters could be slightly ambiguous. The judgment of users are often incorrect through the insertion, deletion or finding the connection between nodes and assigning the category label to every disjoint sub-cluster. The DUFFNN clustering method has prediction success within the prediction of carcinoma patients' survival time, therefore during this work authors design to use this model so as to predict the COV-19 incident globally.

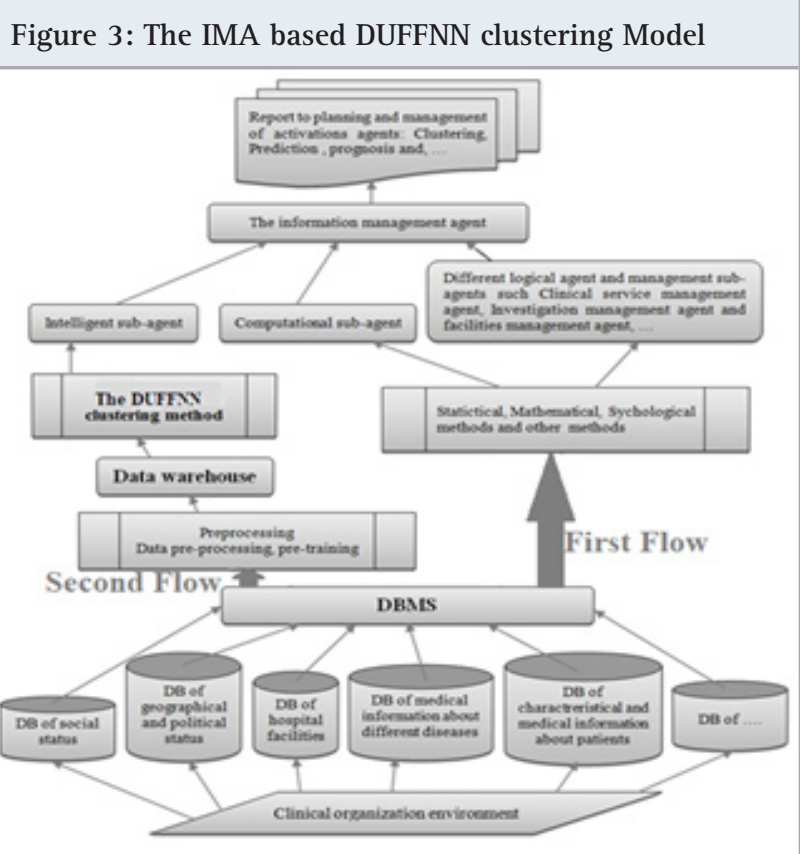

A. Intelligent Multi Agent Systems (IMAS) based on the DUFFNN model: The IMAS with a man-made Intelligent fundamental has great capabilities to be applied in various intents and applications. one among the many topics to use them within the real and virtual areas is to enhance an intelligent model that adopted the machine learning framework to unwrap the entire intricacy of incidence of disease like the COV-19 within the world. Intelligence is common terminologies wont to refer the mind activities, like judging, reasoning , planning and learning. AI is predicated on smart behaviour, and artificial neural network uses it for learning of virtual 
environments. Intelligent Agent (IA) is predicated on the synthetic intelligence properties. Wooldridge in and Langseth enlightened several features of the IA, which are capable of flexible autonomous action to satisfy their design purposes, as follow:

Reactivity: IA obtains data of its area by its sensors, changes internal design objectives of its structure and has suitable actions with feedback periodically.

Pro-activeness: IA is in a position to point out goal absorbed behaviour by taking the ingenuity, responding to variations in their area so as to satisfy their design objectives.

Sociability: IA can cooperate with other agents for compromise and/or collaboration to satisfy their design objectives. Other properties of the IA are self-analysis, learning, adapting and improving through interaction with the world.

Figure 4: Newly Infected vs. Newly Recovered COV-19 as on 28th March,2021 (Src : worldometer/Covidvirus)

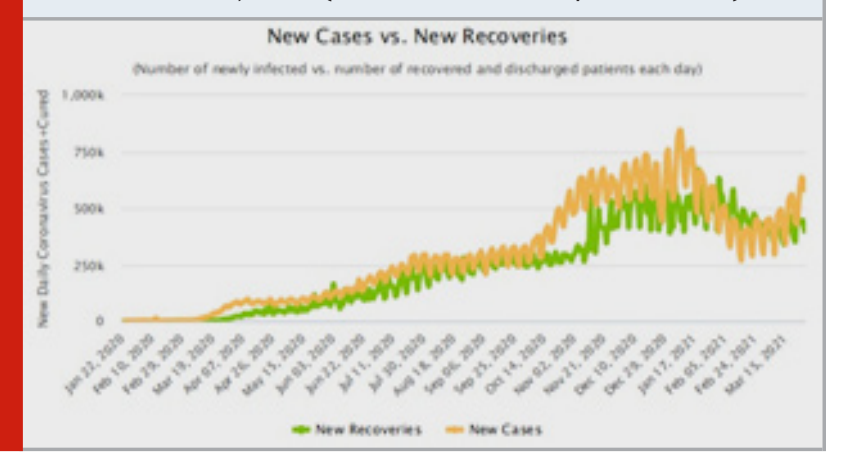

Figure 5: The DUFFNN clustering Model flowchart

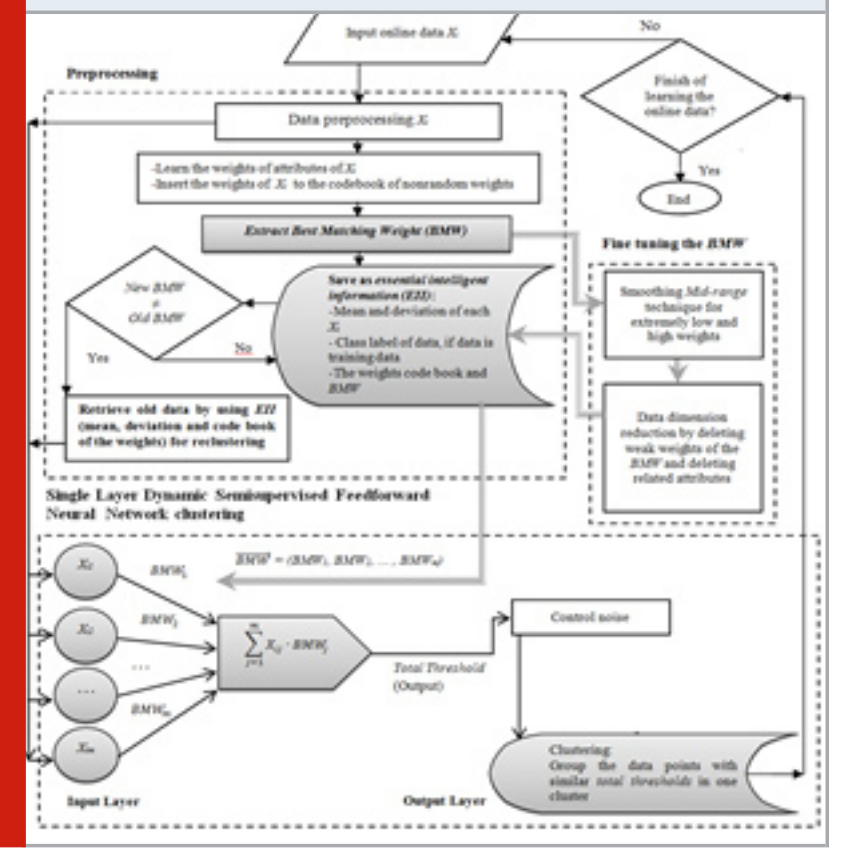

Smart systems apply the multi agent technology to unravel the issues of study of intricate systems and smart management activities. The IMAS based learning combine collection of data from their area, recognition data, intelligent cluster data and prediction future data, selforganization, storage data, delivery data to knowledge management systems like Decision network (DSS) and Management data system (MIS). Padghan and Winikopff explained in the structure of multi agent system is usually covers the Actions, Percepts, Events, Goals, Beliefs, Plans, Messages and Protocols. Yoav \&t Kevin in and Michael clarified about agent based system and intelligent agent as two relevant streams and therefore the gap of a combined framework for blending these two. Bobek and Perko in presented that intelligent agents that are commonly applied in Intelligent Acquisition, Intelligent modelling and Intelligent delivery.

Presently, there's the shortage of a united framework for combination of the two applicable flows of intelligent multi agent technology and learning in real environment. So as to unravel the gap, experiment to think about DUFFNN clustering model as intelligent core of intelligent multi agent based online learning framework. The framework earns the knowledge from the respective environment and its behaviour are often recognized by the weights.

B. The Corona Virus Disease 2019 (C19): In this section, we review on the newest existing diagnostic techniques and treatment of the deadly pandemic. The Corona Virus Disease 2019 or since it is otherwise called COVID -19 may be a deadly viral occurrence that broke call at Wuhan city, China in December 2019. It's been classified under Severe Acute Respiratory Syndrome corona virus 2 or Sarbecovirus (SARS-2). The arrival of SARS-2 is taken into account because the third outcome of an exceedingly zootic corona virus that has been affecting the human population next to the acute acute respiratory syndrome corona virus (SARS) and therefore the Middle East Respiration Syndrome corona virus or Merbecovirus (MERS) within the twenty-first century. With the already prevailing knowledge of SARS and MERS infections, this review are often helpful in imparting novel insights and possible therapeutic objectives for combating the SARS contamination.

Figure 6: The current status of the COV-19 on 28.03.2021

in world countries map based on WHO report

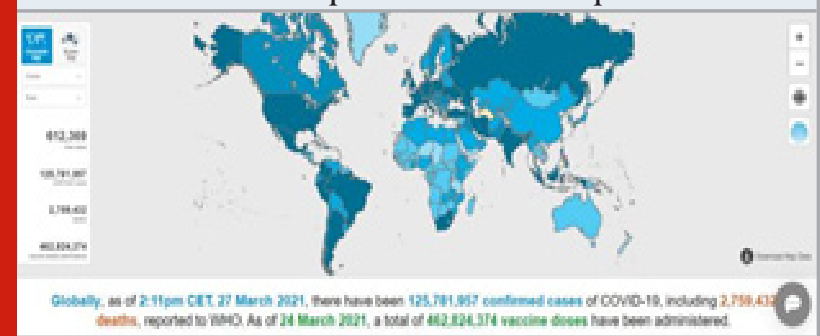

Further, the disease being zootic, it's believed that bats could be the possible favorite reservoir. The transmission routes to humans aren't yet known. There are not any clinically authorized vaccines or antiviral drugs that are 
currently available to be used against COVID -19 and pretty variety of attempts are being made with existing drugs like remdesivir, while few of the countries have come up with vaccines and trials were made with different scenarios of possible treatment with no confirmation on the effectiveness of the drugs, however the infection rate remains not under the control. Corona viruses are most of the world's leading transmittable ailment zoonotic pandemic which may be seen in people and also in animals along side a spread of scientific capabilities from asymptomatic path to fever, cough, gastrointestinal, hepatic, neurologic systems and importantly inflicting infections in breathing that needs to be admitted in ICU at hospitals. These viruses weren't into account as fairly pathogenic for humans till they were visible with the severe acute respiratory syndrome (SARS) at the Guangdong state of China, where it broke out for the primary time in 2002 and 2003.

Figure 7: The number of cases of COV-19 per day in the global world by WHO

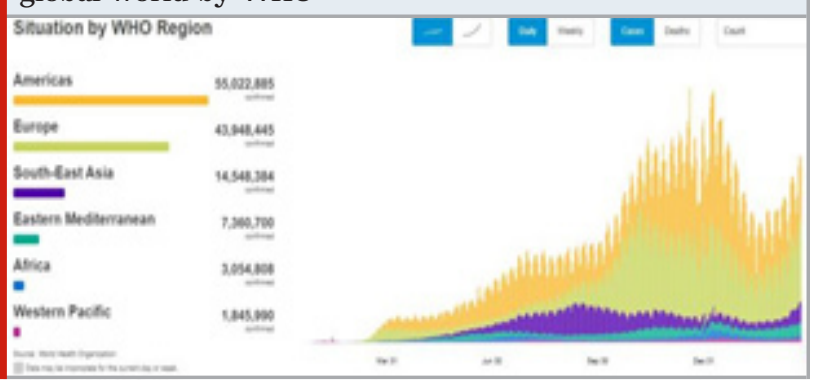

Figure 8: The final BMW weight vector of the global dataset by the DUFFNN method

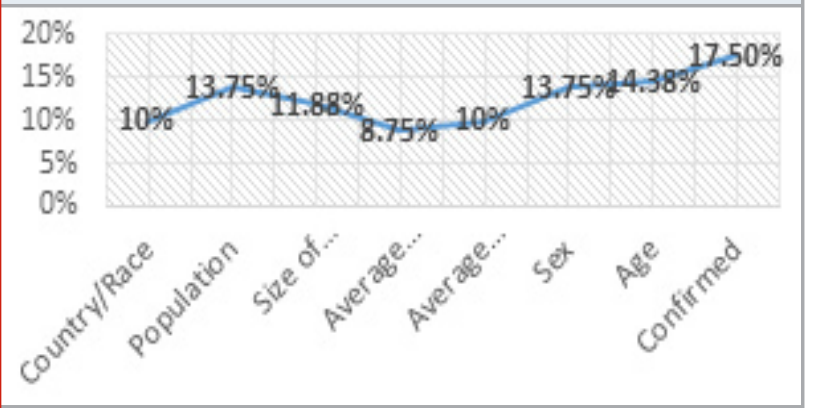

Table 2. Comparison the clustering results on the global data points by the DUFFNN, DSFFNN and related methods

\begin{tabular}{|l|c|c|c|}
\hline $\begin{array}{l}\text { The clustering } \\
\text { method }\end{array}$ & $\begin{array}{c}\text { F-measure } \\
\%\end{array}$ & Epoch & $\begin{array}{c}\text { CPU TIME } \\
\text { (milliseconds) }\end{array}$ \\
\hline DSOM & 71.22 & 800 & 5', 30" and 106 \\
\hline ESOM & 93.04 & 1 & 3" and 102 \\
\hline Semi-ESOM & 95.13 & 1 & 3" and 102 \\
\hline DUFFNN & 98.71 & 1 & 16.80 \\
\hline DSFFNN & 99.82 & 1 & 16.80 \\
\hline
\end{tabular}

On before the above outbreaks, there had been the 2 most known sorts of case as SARS and MERS that have normally precipitated moderate infections in humans with an adequate system. Nearly ten years from it, SARS-2 this point, the opposite type notably pathogenic infected the humans in mass. At first, an unknown pneumonia case became detected on December 12, 2019. This virus was named as COVID - 19 by WHO on 12th January 2019. As of 26nd August, 2020, a complete of 23,518,343 showed infected and 810,492 deaths are taken place as per the updates of WHO. While still the route of transmission to human is under speculation, it's believed to possess been transmitted possibly as zoonotic agent (from animal to human). Subsequent level of transmission from humans to humans was confirmed in Wuhan city after closing the market and evacuation of the cases in China. New cases of COV-19 spreading happened, primarily in several Asian nations and in many nations inclusive of the non Asian also as USA and France. Though the medical facilities have drastically improved now technically and clinically, the death-rate of COVID 19 is high. the sole available precaution to flee from this infection is social distancing, continuous usage of masks and sanitizing the touch.

The two outbreaks of SARS (SARS-e and SARS-2) were first found in Wuhan of China and both are believed to possess originated from the wild animal markets. Scientists studied the Wuhan corona virus ordering to different viruses and observed samples closely shows the similarities of bat corona virus. Scientists partially conclude that the corona virus inflicting SARS originated from a reservoir in bats. Within the case of this new outbreak, it had been traced and now-shuttered Huanan Seafood Wholesale Market in Wuhan, where several wild animals, along side raccoon dogs and snakes, were purchasable . the sole thanks to make sure, where the virus source from is to require samples of DNA from animals that are sold thereon market and from wild snakes and bats therein location. In 2003, SARS first became mentioned as a definite corona virus strain. The origin of the virus not clear, albeit the primary human infections are often traced returned to Guangdong in 2002.

The SARS-e virus spread to quite 8,000 influenza-like ailment infections in 26 countries with almost 800 deaths. The Wuhan corona virus has caused fear everywhere the planet. There are matters that aren't unusual among the corona virus spreading in China and therefore the 2003 SARS/MERS outbreak both belong to the corona virus family and every had been probably handed from animals to people on a wet marketplace. Corona viruses are zoonotic sicknesses which mean they unfold from animals to citizenry. Since wet markets put humans and animals-dogs, chickens, pigs, rats, civets, and more-in near touch, making loop for an epidemic to spread in wholesome. Bats had been the authentic hosts of SARS, and doubtless this corona virus outbreak too. Bats and birds are considered pandemic- ability reservoir species for viruses. The animals were the first supply of Ebola; 
in 1976 it's killed 13,500 citizenry in additional than one outbreak.

To many, the contemporary epidemic sounds similar in 2003 while severe acute respiration syndrome (SARS-e) spread across the country, infecting quite 8,000 citizenry and killing 774 worldwide. However, at an equivalent time as an identical virus triggers the Wuhan corona virus after 10 years later and that they could also be not precisely the similar on. Consistent with literature, early cases of COV-19 had been detected, with the people connected to the Huanan seafood and animal marketplace. In Wuhan 27 people were said to be affected, infected were the likely source of the fresh virus could are from animal. Etiologic investigations had been accomplished in sufferers who administered to the checkup due to similar virus infection.

The weird records of excessive-risk of animal contact within the clinical histories of these sufferers have reinforced the likelihood of an infection moved from animals to humans. On January 22, 2020, novel COVID -19 has been declared to be emerged from wild bats and belonged to the beta corona viruses under group 2 that has Severe Acute Respiratory Syndrome Associated Corona Virus (SARS). Although COV-19 and SARS-e belong to the beta corona virus subgroup, similarity at genome degree is nearly $70 \%$, and there exists a completely unique genetic organization that has been found to show genetic variations from SARS. Almost like the SARS epidemic, this outbreak in China has occurred in spring festival season, which is one among the famous traditional happening in China, for the duration of which nearly million people travel country wide. These favorable situations caused the transmission of this incredibly contagion thereby resulting in severe problems in prevention and control of the epidemic.

The duration of the Spring Festival of China was between January 17 and February 23 in 2003, when the SARS epidemic peaked, and this point the duration of infection peak was found between January 10 and February 18 in 2020. Wuhan, with an honest number of populations is that the center of the epidemic and also an important epicenter within the spring festival transportation network. The estimated sort of vacationers within the course of the 2020-2021 spring pageant has raised 1.7 folds whilst compared with the number traveled in 2003. This large-scale travel site with an enormous number of tourists has also created favorable conditions for the spread this difficult-to-control ailment. The Figure 1 shows the Epidemic curve of confirmed COV-19, by WHO region until 28th March, 2021, where it clearly specifics USA is majorly effected area even today after vaccination campaigns been held world wide.

C. Characteristics of COVID 19: Corono viruses contain RNA as genetic materials and importantly every COV generally has crown like structure with peplomers with 80-160nM in Size and 27-32 kb positive polarity. It contains precise genes in ORF1which encode proteins for viral replication. The peplomers which are present outside the surface of the viruses is solely responsible for the attack and entry into host cells. The receptorbinding domain (RBD) isn't tightly attached among virus, therefore, the virus may additionally infect quite one hosts. Due to its high mutation the recombination rates also are too high. Individuals suffering from COV-19 are found to experience fever, cough, myalgia, fatigue, dyspnea, regular/ reduced leukocyte counts, and radiographic proof of pneumonia, which coincide with the symptoms of SARS-e and MERS-e infections. Hence, although the pathogenesis of COV-19 is poorly understood, an equivalent procedures adopted for SARS-e and MERS-e nevertheless can give us a lot of records on the pathogenesis of SARS-2 infections to facilitate treatment methodologies.

D. Transmission of COV-19: Corona viruses belong to relatives of viruses commonly located in various specific animal species, consisting camels, goats, cats, and bats. Rarely can animal corona viruses attack citizenry, but when humans are attacked, they need led to MERS, SARS within the past, and now to COV-19. Most respiratory viruses are spread through sneezing and coughing. Although the Chinese authorities turned down the chance of human-to human transmission at the start, substantial and sustained transmission among humans has now come to be apparent. Chinese scientists have recommended that a couple of infected humans may transmit the virus to others even before they develop contamination or experience any signs, causing an asymptomatic spread of the virus.

They need been found to spread among people that are in very close contact (approximately 2 feet). It's further noticed that spreading happens typically through respiratory droplets created while an infected person coughs or sneezes, almost like how influenza and different respiratory pathogens spread. Such droplets may additionally land in nearby humans' mouths or noses, or probably be inhaled into the lungs. Whether an individual can get COV-19 by way of touching a floor or object with the virus thereon, after which touching their own mouth, nose, or likely eyes, is currently unclear. Many viruses (which include measles) are notably contagious, at an equivalent time as different viruses are much less so. There are several investigations currently being administered to review the transmissiblity, frequency, and other characteristics associated with COV-19.

\section{The Framework Of Intelligent Multi Agent System} Based The Duffnn Clustering Model: In this section, we propose an IMAS supported the newest efficient ODFFNN model that we believe it's great potentials to use during this area to detect the present status of the COV-19 within the world, so as to predict the longer term and stop. Our IMAS framework has two flows. First flow includes earning knowledge of the prevailing symptoms, diagnostic techniques and treatment of the deadly pandemic of the COV-19. Second flow includes detection of the amount of patient cases, risk of the disease and therefore the polluted spots that they will help us to watch and control the Covid19 risk. 
During this phase, we compare the DUFFNN model with several strong ODUFFNN clustering so as to seek out the simplest efficient model during this area. Therefore, by using our intelligent framework, we discover hot polluted spots within the world, then manage the danger and parameters of the spread of the COV-19, and eventually we predict the longer term situation of this disease. Authors plan to solve the gap of lacking in one united framework for combination of two relevant flows which are IMA systems in world and learning systems. There are numerous topics during this system like intelligent acquisition, intelligent modelling and intelligent delivery. Figure 3 shows plan of intelligent multi agent based neural network clustering system.

Considering the info from everywhere the countries of the planet together health network. The proposed work plan to analyze and style the cluster the planet countries data. The countries data cluster to seek out hot polluted spots. The experiment to manage the danger of infection and finding the effective parameters, features of the symptoms and their weights. The traditional chart has several demerits in managing, data and earning the specified outputs like data-redundancy, data-concurrency while updating the database. Hence the traditional chart to be modified consistent with the IMAS based the DUFFNN. The management information agent gathers the info from area. Generally, the info is distributed and a system with several branches for collecting data is compulsory. Figure 4 shows the small print of the IMA based the DUFFNN clustering system.

The data collected in one source of database and clustered by IMA based efficient DUFFNN clustering model. The very fact data of background is stored in one database, and is controlled by the info Base Management System (DBMS) thru the DBA (Data Base Administrator). The IMAS clusters cases and predicts their states, supported different aims of the system and selected attributes, by sub-agents of the management information agent. The management IA communicates the reports of data to plan and manage the activation of the agent for applying in DSS, MIS, statistical and scientific system software, and other data management systems. The planning and rules to be updated and advanced reports are generated for the confirmation and action authorization. The Intelligent delivery generation of the advanced reporting supported experts opinions by using management and decision systems like MIS and DSS, and consequently ability to proactively reply to special events, to make a decision to update rules and plans. The IMAS has feedback for updating believes and rules too.

The First Flow in order to Earning Knowledge of the COV-19: Considering the case study, within the first flow of the IMAS, the proposed work intended to review and analyze the COV-19 disease and therefore the refore the earned knowledge be saved and the data of the system are going to be updated. The second flow is employed and update the info from this phase too. The novel corona virus emerged from the Hunan seafood market at Wuhan,
China during which bats, snakes, raccoon dogs, palm civets, and different animals are sold. There need to be a whole ban on utilizing wild animals and birds as a supply of food. Human-to-human transmission is occurring, which suggests that more COV-19 instances are predicted to be reported within the future, which may potentially purpose disruptions of world public health structures and economic losses. Abeit availability of vaccines in most of the developed countries are promising clinical trials and prevention strategies are developed fight against human infected corona viruses.

However, the rise rate of infected and mortality are still found from the remainder of the worlds, hence researchers are striving to develop efficient therapeutic techniques to deal with the novel corona viruses. Remdesivir, Lopinavir/ritonavir, Ribavirin, Chloroquine phosphate and Arbidol consequently as per the WHO report from 58 countries are making the come up the reliable vaccines and significantly blocked the COV-19 contamination in infected patients. While the selection of bringing the residents from infected place by numerous nations and bad screening of passengers, have clothed to be the leading reason of unfolding virus in others nations.

There are many agencies working for the event of powerful SARS-CoV-2 drug and vaccinating the possible. However, there's a requirement for quick human and animal-primarily based trails as those vaccines still require few months for commercialization, which recently happening with covaxin in India. Besides the event of green drug, a way to diagnose SARS-CoV-2 in suspected patient is additionally required. Therefore, an accurate and rapid diagnostic package or meter for detection of SARS-CoV-2 in suspected patients is required, because the PCR based testing is high-priced and time consuming. The therapeutic techniques utilized by China people, must even be employed by other countries to regulate the pandemic disease as a prevention of the further infection.

\section{Methods to Detect COV-19:}

Nucleic acid detection or RT-qPCR: Real Time qualitative Polymerase chain reaction (RT- qPCR) and highthroughput sequencing are the foremost efficiently used technologies to spot COV-19 in real-time. The assured COV-19 entity approach for SARS-2 is virus blood culture and high-throughput sequencing of the entire genome. The software excessive-throughput sequencing techniques in diagnosis are only a few thanks to its excessive cost. RT-qPCR is that the maximum used powerful and direct technique for detecting pathogenic viruses in respiratory secretions and blood. RT-qPCR proves and also confirmed high responsiveness and specificity for SARS and MERS contamination. Thus, it's vital to reinforce the detection of RT-qPCR for SARS-2 contamination sensitivity.

High Throughput sequencing: The MagEx STARlet assay ready computer permits excessive-throughput magnetic bead-based totall RNA extraction of biological samples 
The PCR (polymerase chain reaction) assay prepared computer is pre-configured and qualified to automate excessive-throughput pre-PCR sample setup using the today's Centers for Disease Control and Prevention (CDC) protocols. Both assay equipped workstations are designed to figure with any provider's assay chemistry, like those supposed for qualitative detection of the corona virus.

ELISA, An enzyme-linked immunosorbent assay, also called ELISA or EIA, may be a make sure detects and analyzes antibodies within the blood. Currently, realtime polymerase chain reaction (RT-PCR) assessments genetic fabric to hold out corona virus testing. However, those assessments have low costs of specificity and sensitivity and pose dangers related to specimen series and sample dealing. POCT of IgM/IgG is one among the world's first rapid point-of-care Test (POCT) lateral float immunoassays for the analysis of corona viral infection . It also can be wont to check human serum or plasma samples. This clinical examination are often used for fast screening of the virus which may be symptomatic or asymptomatic. CT Scans is one essential supplementary diagnostic procedure. For an individual with an excessive scientific intuition of SARS-2 contamination with positive RT-qPCR result, a mix of frequent chest CT scan and RT-qPCR tests probably be beneficial. Specially the high resolution CT (HRCT) for the chest is vital for an early diagnosis and assessment of severity of the ailment in people infected with SARS-2. Several researches have examined chest CT images of patients infected with SARS.

The standard CT photos show bilateral pulmonary opacities, often with a rounded morphology and a peripheral lung parenchyma distribution. Peripheral predominance within the lungs is clearly visible in people infected with SARS-e and MERS-e infections, and therefore the same is observed within the case of SARS-2 contamination. However, the CT scan technology might not serve the aim in majority of the cases emerging from a highly prevalent area thanks to its high cost. Further, CT scans additionally have some shortcomings, which include indistinguishability from different viral infection and therefore the hysteresis obtained of ordinary CT imaging. Given the shortcomings of the presently used macromolecule detection and CT scans for the analysis of COV-19, clinical laboratories need to apply some immunological detection kits that specialize in viral antigens or antibodies as quickly as viable. Currently, POCT of IgM/IgG and ELISA kits for SARS-2 had been evolved and pre-tested by means of some groups and have proven higher detection fees than macromolecule detection, but there's still no product or posted article.

C. Prevention: Hygiene and Cleaning - The First Defence: Even though presently lesser availability of the vaccine to the remainder of the planet and handy to fore stall a contamination with COV-19 there are not any control over the infection rate. The satisfactory thanks to safeguard from infection is to forestall the virus being released. However, CDC (Center of Disease Control) additionally recommends ordinary defensive measures to save lots of from respiratory viruses with. it's suggested to avoid near contact with sick people, reduce contact with lips, nostril and mouth with unwashed fingers and a number of other precautionary safety measures are being widely given awareness to follow. There's no approved clear antiviral treatment for an infection of COV-19. People infected with COV-19 will obtain medical remedy to help in symptom relief. Treatment for severe cases includes remedy to take care of essential functions of the organ. This guidance is meant for persons with showed or suspected COV-19 contamination, along side those who don't got to be hospitalized and who can also obtain care reception. Below are prevention measures for people with confirmed or suspected COV-19 infection, who don't got to be hospitalized and individuals with COV-19 contamination who've been hospitalized and who are considered to be medically safe to travel domestic after discharging. The physicians and public doctors are deciding whether the infected could also be handled reception.

D. Current treatment techniques for COV-19: Similar to SARS-e and MERS-e, there are currently no medically proven desired antiviral agents to be administered for SARS-2 infection. The supportive remedy, which includes oxygen remedy, conservation fluid management, and the use of broad-spectrum antibiotics to cowl secondary bacterial infection, remains to be the maximum essential management method. There are 92 vaccines and 58 new, 45 antibodies, 20 Anti-viral, 12 cell-based therapies, 5 RNA-based treatment and 52 other solutions currently being explored to fight the new corona virus SARSCoV-2. The latest graph is shown in Fig 4 the newly infected verses newly recovered.

Further, Viral focused inhibitors: Remdesivir, an adenosine analogue which will aim the RNA-dependent RNA polymerase and block viral RNA synthesis, which has been a promising antiviral convict to an enormous selection of RNA viruses (consisting of SARS/MERS-e) infections in cultured cells, mice and nonhuman primate models. Remdesivir and chloroquine had been established to inhibit SARS-2 correctly in vitro. Hence, other nucleoside analogues, consisting of favipiravir, ribavirin and galidesivir are often doubtlessly clinically relevant con to SARS-2. Chymotrypsin-like (3C-like protease, 3CLpro) and papain-like protease (PLP) are non-structural proteins, which have an important feature for corona viral replication and may inhibit the host innate immune responses. The 3CLpro inhibitors, like cinanserin and flavonoids, and PLP inhibitors, inclusive of diarylheptanoids, are different attractive selections to combat against SARS-2.

Antibody and plasma remedy has also been trialed and reported that there are numerous convalescent sufferers donating plasma con to SARS-2, simply as SARS-e and MERS-e trials. A SARS corona virus-specific human antibody can bind potently with the receptor-binding domain (RBD) of SARS-2 and has the potential to be evolved as candidate therapeutics of SARS infections. There are several vaccination techniques towards SARS-e, 
MERS-e tested in animals, including a stay-attenuated virus, viral vectors, inactivated virus, subunit vaccines, recombinant deoxyribonucleic acid, and proteins vaccines. Several new pharmaceutical drugs, which include HIV tablets and stem cells, had been testified in a number of the scientific trials. However, the threat of the second or the third wave is predicted and still there are new infection and deaths are being recorded worldwide. The doctors are being in search of the post vaccine study and effect of revolutionized effect of the virus.

E. The Second Flow based on the DUFFNN Model as the Intelligent Core: The second phase of the framework designed to apply to handle data, rules, plans, and future prediction. Fig 6 shows the detail of the DUFFNN clustering method:

Pre-processing: The DUFFNN method considers a neighborhood of the memory called Essential Important Information (EII) and initializes the EII by learning and storing the mean, deviation and non-random weights of every online input file without storing any input file within the memory. The code book of weights is completed by training online data supported the load Linear Analysis (WLA) technique. Accordingly, the unique BMW vector is mined from the code book of the weights and stored because the EII. WLA pre-processing may be a combination of knowledge pre-processing of the web input file so as to think about the input value within the range $(0,1)$ and cleaning the info like MinMax technique; and pre-training by computing BMW. the quality Gaussian distribution (SND) and therefore the Best Match Weight (BMW) vector generate the mean vector of the code book of the non-random weights . Fine tuning process refers to modifying the weights accurately so as to succeed better results of clustering the input file, includes smoothing the weights and pruning the weak weights so as to dimension reduction.

The topology of single layer DUFFNN includes that the amount of layers and nodes are clear which contains of just an input layer with $n$ nodes equal the amount of attributes of input file and an output layer with just one node. The units of the input layer are fed by the traditional data values from the info pre-processing stage. When the model obtains the acceptable weights, the input layer is fixed with weights connections to the output layer for learning without updating the weights. Each unit applies a related weight component of the BMW vector. The output layer consists of a unit with a weighted sum function for calculating the particular desired output without using any class label. The training of the DUFFNN is passed just in one epoch and is predicated on non-random weights, with none weight updating, activation function and error function like mean square error. The edge as output is calculated by consuming normal values of the input file and therefore the BMW vector. Thanks to use the mean of the weights for calculating the BMW, the range and properties of the input file values cannot dominate the values of the thresholds. After clustering the info node by the DUFFNN, the proposed method considers the category label as constraint so as to enhance the accuracy of the results of clustering, and alter to the dynamic semi-supervised feedforward neural network (DSFFNN) model.

\section{F. Datasets:}

In this research, we apply several datasets from various sources in order to find effective elements to find the best accuracy results and prediction of the COV-19 incident disease, as follow:

World Health Organization (WHO) with 259 records of global countries.

- https://www.who.int/emergencies/diseases/novelcoronavirus-2019/situation-reports

- National Centers for Environmental Information. Daily data of the average temperature was obtained.

- https://www.ncei.noaa.gov/access/search/datasearch/global-summary-of-the-day

- Natural earth. http://www.naturalearthdata.com/ downloads/110m-cultural-vectors/

- $\quad$ The dataset of COV-19 from Kaggle as "Novel Corona Virus 2019 Dataset”. https://www. kaggle.com/ sudalairajkumar/novel-corona-virus-2019-dataset/ (updated version 147)

- The Humanitarian Data Exchange (HDX). https:// data.humdata.org/dataset/novel-coronavirus-2019ncov-cases (live updated)

For an experimental results, the attributes of population, size of country, average temperature, average humidity, sex, age are used, also the Corona disease cases includes confirmed and death cases that are considered based on global world countries. The dataset includes two classes of infected and death.

III. Experimental Results and Evaluation: IMA based DUFFNN clustering system has capability of predicting future status of patients and spread of the COV-19 disease and showing complex relations between components of systems. Authors mean to use datasets and models mentioned within the previous sections and present the respective results. the present status of the COV-19 on 28.03.2021 in world countries map is as follow in Fig 7; recorded Globally, as of 2:11pm CET, 28 March 2021, there are 125,781,957 confirmed cases of COVID-19, including 2,759,432 deaths, reported to WHO. As of 25 March 2021, a complete of 462,824,374 vaccine doses are administered. Among which the countries within the region of Europe, South-East Asia, Eastern Mediterrenean and Western Pacific found continuously increasing infection rate along side mortality. Following Fig 6 showing the present status of the countries map by WHO.

We have consider the dataset of the Covid19 cases collected by World Health Organization (WHO). This dataset includes 259 records of global countries with the confirmed and deaths cases from 31 January 2020 until 25 March 2021 as shown in Figure 7. For an experimental results, we apply several attributes from different sources as shown within the section 2-3 Datasets. So as 
to implement the DUFFNN, DSFFNN and other related ODUFFNN methods, the primary got to pre-process and clean the info . Compute the clustering accuracy of the DUFFNN on the info points, by using F-measure or balanced F-Score function with 10 folds of the test set. Figure 9 show the ultimate computed weight vector components (BMW) of the received global world dataset by using the DUFFNN clustering method. the info has two class labels: infected and death.

The weight of every attribute shows what proportion the attribute is effective within the output results and explained hence selected few attributes from different datasets that cause the output result's more accurate so as to predict the status of COV-19 within the world countries. As shown within the Fig 8, the effective items considered are population, sex, age and confirmed cases. However, the weights show that race, average of temperature and average of humidity are normal and aren't very effective. For the experiment, authors here during this research compared the results of the DUFFNN and DSFFNN methods with the results of several related ODUFFNN methods. Within the ESOM, by considering the parameter set $\mathrm{P}=\{\varepsilon, \delta, \gamma, \mathrm{Tp}\}, \varepsilon$ as distance threshold, $\delta$ for controlling the spread of the neighborhood, $\gamma$ as a little constant learning rate, $\mathrm{Tp}$ because the steps of learning time, and $\beta$ as forgetting constant, we considered: $\beta=$ $0.8, \varepsilon=0.005, \delta=\varepsilon$ and $\gamma=0.005$. Within the DSOM, the experiment considered that the parameters $\varepsilon$ the speed of learning and $\delta$ control distance from the BMU because the elasticity: $\varepsilon=0.100, \delta=1.25$. Table 2 shows the speed of processing on the idea of the amount of epochs and therefore the accuracy on the idea of the density of the F-measure for the worldwide data points.

Table 2 demonstrates, the DSOM clustered the info points with $71.22 \%$ accuracy by the F-measure after 800 epochs during 5', 30" and 106 milliseconds. The ESOM clustered the info points with $93.04 \%$ accuracy by the F-measure after 1 epoch during 3" and 102 milliseconds. The SemiESOM clustered the info points with $95.13 \%$ accuracy by the F-measure. The DUFFNN clustering method clustered this data set with $98.71 \%$ accuracy by F-measure during 1 epoch and 16.80 milliseconds and DUFFNN clustering method clustered this data set with 98.71\% accuracy. The DSFFNN clusters method clustered with 99.82\%. Fig 9: Comparison of the prediction of the amount of the COV-19 cases per day within the global world by using different current strong ODUFFNN clustering methods Based on the DSFFNN clustering prediction, the map of world for the COVID 19 disease incidence probable be very almost like map in Fig 6 of the WHO report with 99.76\% accuracy. The DSFFNN clustering accuracy are going to be close $100 \%$ when the important values of dataset are reported by the countries.

\section{CONCLUSION}

This research work has successfully proposed an Intelligent Multi Agent Systems supported the DUFFNN model that has two streams. First stream includes earning knowledge of the newest existing symptoms, diagnostic techniques and treatment of the deadly pandemic of the COV-19. Second flow includes detection of the amount of patient cases, risk of the disease and therefore the polluted spots, that they will help us to watch and control the COV-19 risk and parameters. The experimental results show that the DUFFNN and consequently DSFFNN method have the simplest accuracies of $98.99 \%$ and $99.97 \%$, respectively. The DUFFNN method are ready to show the load of every attribute that used during clustering. Therefore, it's possible to seek out an affective items that cause more accurate leads to order to prediction. For future work, authors extending the study to use other attributes and parameters from different sources which will be extract better clustering results with higher accuracy.

\section{REFERENCES}

Asadi, R., Mustapha, N., Sulaiman, N., \&t Shiri, N. (2009). New supervised multi layer feed forward neural network model to accelerate classification with high accuracy. European Journal of Scientific Research, 33(1), 163178.

Asadi, R., A. K., Asadi, M., \& Asadi, S. (2015). A dynamic semisupervised feedforward neural network clustering. Artificial Intelligence for Engineering Design, Analysis and Manufacturing (AIEDAM), May 2016, pp 1 - 25. The Cambridge University Press, University of Southern California, USA.

Asadi, R., A. K., Asadi, M., \&t Asadi, S. (2015). An Unsupervised Feed Forward Neural Network Method for Efficient Clustering. The International Arab Journal of Information Technology (IAJIT), . Zarqa University, Jordan.

Bobek, S. and Perko, I., (2006). Intelligent Agent Based Business Intelligence. University of Maribor, Faculty of Economics and Business, Razlagova ulica14, SI-2000 Maribor, Slovenia.

Bobek, S., Sternad, S. and Perko, I., (2005). Business Performance Models In Management Information Systems.

Bouchachia, A., Gabrys, B., \&t Sahel, Z. (2007). Overview of some incremental learning algorithms.

Brachman, R. J., \&t Anand, T. (1994). The Process of Knowledge Discovery in Databases: A First Sketch. Paper presented at the KDD Workshop.

Chakraborty, R. (2010). Fundamentals of Neural Networks. Soft Computing, 7-14.

David, P., Alan, M. and Randy, G., (1998). Computational Intelligence: A Logical Approach. New York: Oxford University Press. http://www.cs.ubc.ca/spider/poole/ ci.html.

Deng, D., \&t Kasabov, N. (2003). On-line pattern analysis by evolving self-organizing maps. Neurocomputing, 51, 87-103.

Fritzke, B. (1995). A growing neural gas network learns topologies.

Furao, S., Ogura, T., \& Hasegawa, 0. (2007). An enhanced self-organizing incremental neural network for online unsupervised learning. Neural Networks, 20(8), 893- 
903.

Germano, T. (1999). Self Organizing Maps. Accessed at http://davis.wpi.edu/ matt/courses/soms.

Gui, V., Vasiu, R., \&t Bojkovic, Z. (2001). A new operator for image enhancement. Facta universitatis-series: Electronics and Energetics, 14(1), 109-117.

Hamker, F. H. (2001). Life-long learning Cell Structures-continuously learning without catastrophic interference. Neural Networks, 14(4-5), 551-573.

Hebboul, A., Hacini, M., \&t Hachouf, F. (2011a). An incremental parallel neural network for unsupervised classification. Paper presented at the Proc. 7th Int. Workshop on Systems, Signal Processing Systems and Their Applications (WOSSPA).

Hebboul, A., Hacini, M., \&t Hachouf, F. (2011b). An incremental parallel neural network for unsupervised classification.

Hinton, G. E., \& Salakhutdinov, R. R. (2006). Reducing the dimensionality of data with neural networks. Science, 313(5786), 504.

Honkela, T. (1998). Description of Kohonen's SelfOrganizing Map. Accessed at http://www.cis.hut.fi/ tho/ thesis.

Hsu, J. (2003). Data Mining and Business Intelligence: Tools, Technologies. Business Intelligence in the Digital Economy: Opportunities, Limitations and Risks: Opportunities, Limitations and Risks, 141.

Jean, J. S., \& Wang, J. (1994). Weight smoothing to improve network generalization. IEEE Transactions on Neural Networks, 5(5), 752-763.

Kamiya, Y., Ishii, T., Furao, S., \&t Hasegawa, 0. (2007). An online semi-supervised clustering algorithm based on a self-organizing incremental neural network. Paper presented at the Proc. Int. Joint Conf. Neural Networks (IJCNN), IEEE.

Kasabov, N. K. (1998). ECOS: Evolving Connectionist Systems and the ECO Learning Paradigm. Paper presented at the Proc. 5th Int. Conf. Neural Information Processing, ICONIP'98, Kitakyushu, Japan.

Kohonen, T. (2000). Self-Organization Maps (3rd ed.): Berlin: Springer-Verlag.

Kulkarni, P. A., \&t Mulay, P. (2013). Evolve systems using incremental clustering approach. Evolving Systems, 4(2), 71-85.

Langseth, J. and Vivarat, N., (2005). Why Proactive Business Intelligence Is A Hallmark Of The Real-Time Enterprise: Outward Bound. Intelligent enterprise.
Linde, Y., Buzo, A., \&t Gray, R. (1980). An algorithm for vector quantizer design. IEEE Transactions on Communications, 28(1), 84-95.

MacQueen, J. (1967). Some methods for classification and analysis of multivariate observations. Paper presented at the Proceedings of the fifth Berkeley symposium on mathematical statistics and probability.

Martinetz, T. M., Berkovich, S. G., \&t Schulten, K. J. (1993). Neural-gas' network for vector quantization and its application to time-series prediction. IEEE Transactions on Neural Networks, 4(4), 558-569.

Michael W., (2001). An Introduction to Multi agent systems. Department of Computer Science, University of Iiverpool, UK. ISBN 0-471-49691-X.

Nils, N., (1998). Artificial Intelligence: A New Synthesis. Morgan Kaufmann Publishers. ISBN 978-1-55860467-4.

Padghan, L. and Winikopff, M., (2004). Developing Intelligent Agent Systems. Wiley.

Prudent, Y., \&t Ennaji, A. (2005). An incremental growing neural gas learns topologies. Paper presented at the Proc. IEEE Int. Joint Conf. Neural Networks, IJCNN'05.

Rougier, N., \&t Boniface, Y. (2011). Dynamic selforganising map. Neurocomputing, 74(11), 1840-1847.

Shen, F., Yu, H., Sakurai, K., \&t Hasegawa, 0. (2011). An incremental online semi-supervised active learning algorithm based on self-organizing incremental neural network. Neural Computing and Applications, 20(7), 1061-1074.

Stuart J. R. and Peter N., (2003). Artificial Intelligence: A Modern Approach . Second edition, Upper Saddle River, NJ: Prentice Hall, ISBN 0-13-790395-2.

Ultsch, A., \&t Siemon, H. P. (1990). $\{\mathrm{K}\}$ ohonen|'s Self Organizing Feature Maps for Exploratory Data Analysis.

Van der Maaten, L., Postma, E., \&t Van den Herik, H. (2007). Dimensionality reduction: A comparative review. Preprint.

Vijayan S. (2006).Application of agents and Intelligent Information Technologies. ISBN 1-59904-265-7. Published in USA by Idea group publishing.

Wooldridge, M., (2002). An Introduction To Multi Agent Systems. West Sussex, Willey.

Yoav Sh. and Kevin L., (2009). Multi Agent Systems: Algorithmic, Game-Theoretic, and Logical Foundation. First published. Printed in the USA. ISBN 978-0-52189943-7. 